\section{THREE-YEAR OUTCOMES WITH FIRST-LINE PEMBROLIZUMAB FOR METASTATIC NON-SMALL-CELL LUNG CANCER (NSCLC) WITH A VERY HIGH PD-L1 TUMOR PROPORTION SCORE (TPS) $\geq 90 \%$}

${ }^{1}$ Biagio Ricciuti*, 'Joao Victor Alessi, 'Stephanie Alden, 'Giuseppe Lamberti, 'Victor Vaz, ${ }^{1}$ Adriana Barrichello, ${ }^{2}$ Lynette Sholl, ${ }^{1}$ Mark Awad. 'Dana-Farber Cancer Institute, Boston, MA, USA; ${ }^{2}$ Brigham and Women's Hospital, Boston, MA, USA

Background Although 1st-line PD-1 monotherapy has improved survival in patients with advanced NSCLC and a PD-L1 TPS $\geq 50 \%$, responses occur in $\sim 45 \%$ of patients. We have previously shown that among patients with NSCLC and PD-L1 expression of $\geq 50 \%$ treated with 1 st-line pembrolizumab, clinical outcomes are significantly improved in those with a PD-L1 TPS of $\geq 90 \%$. ${ }^{1}$ Here, we report the 3 -year follow-up outcomes to 1 st-line pembrolizumab in patients with a PD-L1 TPS $\geq 90 \%$ vs $<50-89 \%$, and genomic differences between these groups.

Methods Patients with stage IV EGFR/ALK wild type NSCLC and PD-L1 TPS $\geq 50 \%$ who received 1st-line pembrolizumab monotherapy at Dana-Farber Cancer Institute were included. Comprehensive tumor genomic profiling was performed to examine genomic correlates of a very high PD-L1 expression on an expanded cohort of NSCLC samples.

Results Among 150 patients included, median age was 69 (range: 46-92), 55.3\% were women, 91.9\% were current smokers, and $34.0 \%$ had a KRAS mutation. At a median follow-up of 38.5 months, median progression-free (mPFS) and overall survival (mOS) in the entire cohort were 4.8 months, and 20.0 months, respectively. When compared to patients with a PD-L1 expression of 50-89\% $(\mathrm{N}=81)$, those with PDL1 TPS $\geq 90 \%(\mathrm{~N}=69)$ had a significantly longer mPFS (6.0 vs 3.9 months, HR $0.66, \mathrm{P}=0.02$ ), and longer $\operatorname{mOS}(27.0$ vs 14.6 months, HR $0.63, \mathrm{P}=0.03$; figure 1 ). Kaplan-Meier estimates of the 3-year PFS and OS were $23.0 \%$ and $43.2 \%$ in the PD-L1 TPS $\geq 90 \%$ groups, and $8.6 \%$ and $24.0 \%$ in the PD-L1 TPS 50-89\% group, respectively. A PD-L1 TPS $\geq 90 \%$ was confirmed to be an independent predictor of improved PFS (HR 0.58, P=0.02) and OS (HR 0.57, P=0.01) at multivariable analysis. Patients whose tumors had a PD-L1 TPS $\geq 90 \%$ were also significantly more likely to complete 35 cycles of therapy compared to those with PD-L1 TPS of 50$89 \%(17.4 \%$ vs $3.7 \%, \mathrm{P}<0.01$, figure 2$)$. Tumor genomic profiling from 500 NSCLC samples revealed that mutations in STK11, KEAP1, FBXW7, and CTNNB1, which have been previously correlated with immunotherapy resistance, were significantly enriched tumors with a PD-L1 TPS of 50-89\% compared to those with a PD-L1 TPS $\geq 90 \%$ (figure 3 ).
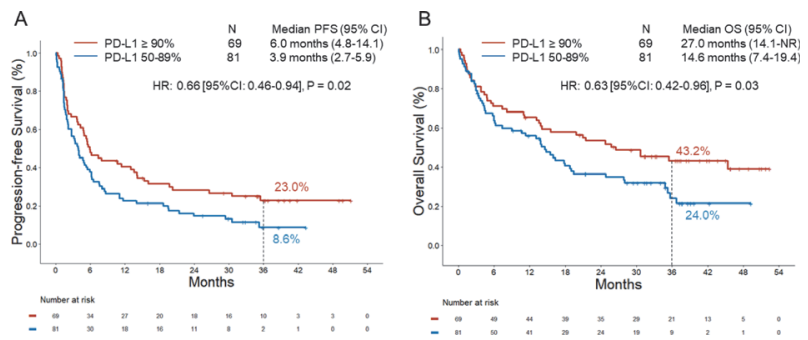

Abstract 312 Figure 1 (A) Progression-free and (B) overall survival to 1st line pembrolizumab among patients with PD-L1 TPS $\geq 90 \%$ vs 50 $89 \%$, at a 3-year follow-up.

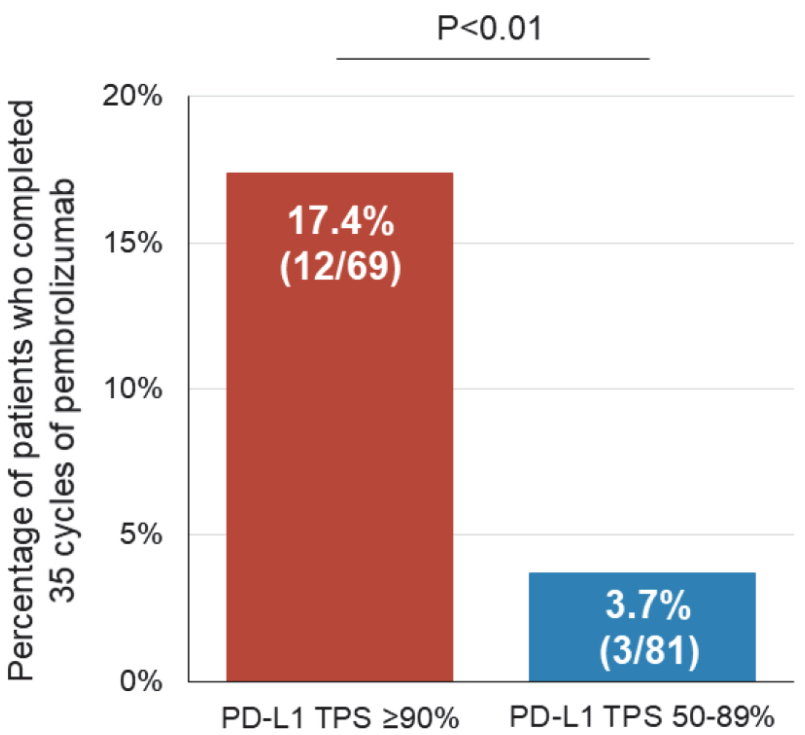

Abstract 312 Figure 2 Barplot showing the percentage of patients who completed 35 cycles of pembrolizumab monotherapy in the PD-L1 TPS $\geq 90 \%$ and $50-89 \%$ groups
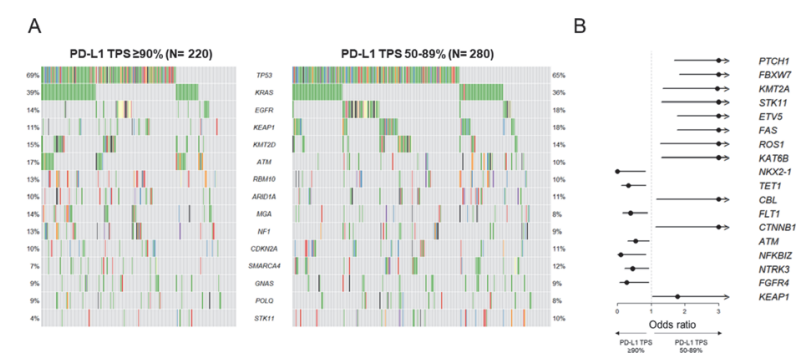

Abstract 312 Figure 3 (A) Oncoprint plot showing the top 15 genes mutated in NSCLC with PD-L1 TPS $\geq 90 \%$ and $50-89 \%$. (B) Gene mutation enrichment analysis showing differentially mutated genes between NSCLCs with PD-L1 TPS $\geq 90 \%$ and $50-89 \%$. * * Adjusted P value $<0.01$; ${ }^{*}$ Adjusted $P$ value $<0.05$.

Conclusions Pembrolizumab monotherapy continues to demonstrate a meaningful long-term survival benefit at a 3-year follow-up in patients with advanced NSCLC and a PD-L1 TPS $\geq 90 \%$ vs $50-89 \%$. NSCLCs with very high PD-L1 TPS have a more favorable genomic profile. These findings have implications for treatment selection and for clinical trial interpretation and design.

\section{REFERENCE}

1. . Aguilar EJ, Ricciuti B, Gainor JF, et al. Outcomes to first-line pembrolizumab in patients with non-small-cell lung cancer and very high PD-L1 expression. Ann Oncol 2019 October 1;30(10):1653-1659

http://dx.doi.org/10.1136/jitc-2021-SITC2021.312 